\title{
The value of exfoliative cell cytology in the diagnosis of exudative pleural effusions
}

\author{
Cuneyt Tetikkurt1, Nail Yılmaz1, Seza Tetikkurt², Sule Gundogdu', Rian Disci \\ 1 Pulmonary Diseases Department, Cerrahpasa Medical Faculty, Istanbul University \\ 2 Pathology Department, Bağcılar Training and Research Hospital, University of Health Sciences, Istanbul \\ 3 Department of Biostatistics and Medical Informatics, Istanbul Medical Faculty, Istanbul University, Turkey
}

\begin{abstract}
The sensitivity and specificity of exfoliative cell cytology for the diagnosis of exudative pleural effusions varies widely according to the etiologic causes. The aim of this study is to assess the diagnostic value of exfoliative cell cytology for the identification of exudative pleural effusions. This is a retrospective study of the patients with an exudative pleural effusion admitted at our clinic in the last twenty years. We have conducted the clinical, the cytological findings, and the diagnostic results of six hundred patients from hospital records. Male to female ratio was 2.2:1 with a mean age of 42.8 years (range 18-78 years) among the patients. Samples were processed and evaluated according to the standard methods. Cytology results were reviewed and the patients were stratified according to the final diagnosis of their disease. Of the six hundred exudative effusions, 240 were malignant on exfoliative cytology pleural fluid alone. Adenocarcinoma was the most common type of malignancy. Tuberculosis was the second most frequent etiology for the exudative effusions followed by infection and collagen vascular diseases. Diagnostic accuracy of cytology showed a good correlation with
\end{abstract}

Corresponding author: Dr. Cuneyt Tetikkurt, Pulmonary Diseases Department, Cerrahpasa Medical Faculty, Istanbul University, Tanzimat sokak, Serkan Apt. No 8/16, Caddebostan, 34728 Istanbul, Turkey. Tel. $+90.216 .3601977,+90.216 .3596035$ - Fax: +90.212 .5870217 .

E-mail: tetikkurt@gmail.com

Key words: Exudative pleural effusion; cytology; pleural effusion cytology; exfoliative cytology.

Contributions: CT, study design, manuscript writing; NY, pleural effusion cytology evaluation; ST, pathologic data evaluation, writing of the manuscript's pathology section; SG, contribution to patient data analysis and patient follow up; RD, statistical analysis.

Conflict of interest: The Authors declare no conflict of interest.

Received for publication: 17 April 2018

Accepted for publication: 1 August 2018

CCopyright C. Tetikkurt et al., 2018

Tipografia PI-ME Editrice, Italy

Monaldi Archives for Chest Disease 2018; 88:944

doi: 10.4081/monaldi.2018.944

This article is distributed under the terms of the Creative Commons Attribution Noncommercial License (by-nc 4.0) which permits any noncommercial use, distribution, and reproduction in any medium, provided the original author(s) and source are credited. the final diagnosis with an overall $70.1 \%$ sensitivity, $62.5 \%$ specificity, and a $95.9 \%$ positive predictive value for all exudative pleural effusions. Cytologic examination of the pleural fluid is a simple non-invasive procedure as the initial step for the diagnostic work up of patients with a pleural effusion. Exfoliative cytology provides high a final diagnostic yield for the identification of an exudative pleural effusion etiology. Furthermore, cytologic analysis leads the clinician into the correct diagnostic pathway as the most informative laboratory tool even when it was not diagnostic by itself for equivocal cases.

\section{Introduction}

Exfoliative pleural effusion cytology is a simple and a non-invasive technique for the identification of exudative pleural effusion etiology. The diagnostic yield of pleural fluid cytology is attributable to the fact that the cell population present in the sediment represents a much larger surface area than that obtained by biopsy [1]. Determining the cause of a pleural effusion is significantly facilitated by the cytologic examination of the pleural fluid [2]. Cell cytology may identify the etiology, facilitates the clinician to proceed in the correct diagnostic pathway establishing the differential diagnosis, may provide useful clinical information for treatment, and the prognostic outcome in patients presenting with an exudative pleural effusion. For malignant effusions, exfoliative cytology may be a fast, an efficient, and a minimally invasive clinical tool for the final diagnosis. Almost all adenocarcinomas are diagnosed by cytologic examination [3,4]. Exfoliative cell cytology may also provide crucial data for the identification of exudative effusions [2]. This study was carried out to determine the diagnostic yield of pleural fluid cytology alone as the initial step for the final diagnosis of exudative pleural effusions. The other aim was to evaluate the sensitivity and the specificity of exfoliative cytology if it was collaborated with the other clinical, radiologic, and laboratory results. We have also analyzed the utility of cytology and differential cell count in the diagnostic pathway of exudative pleural effusions in equivocal cases.

\section{Materials and Methods}

Differential cell count and exfoliative cytology findings from the exudative pleural effusions from six hundred patients admitted during January 1998 and April 2018 at our clinic were evaluated retrospectively. The study has been approved by the IRB/Ethics Committee of Cerrahpasa Medical Faculty. A routine blood biochemistry analysis with complete blood count was performed simultaneously with the thoracentesis. Pleural fluid was analyzed for protein, LDH, albumin, polymorphonuclear leukocytes, and differential cell count. Light's criteria and serum pleural fluid albumin difference was used to separate exudates from transudates while pleural cholesterol level was utilized in 
equivocal cases. Chest $\mathrm{x}$-ray and computed tomography of the thorax were performed in every subject. Consent was taken from all patients in whom thoracentesis, closed or thoracoscopic pleural, bronchoscopic, mediastinal or peripheral lymph node, and any other organ biopsy was done for the histopathologic tissue sampling. Bronchoscopy was performed in cases with pulmonary radiologic findings. The patients were placed in the sitting position for thoracentesis or closed pleural biopsy. A 22-gauge needle filled with $2 \mathrm{ml} 2 \%$ xylocaine was inserted through the intercostal space into the subcutaneous and intercostal tissues while periodically injecting small amounts of xylocaine and aspirating fluid. Three samples of ten milliliters pleural fluid were collected in sterile vials. The samples were sent for biochemistry, cytology, and microbiology evaluation. If the initial sample was not diagnostic a second or a third sample was sent for cytologic evaluation. Abrams needle was used for closed pleural biopsy and four samples of parietal pleural tissue were taken at different sites. The pathologic sections were stained with hematoxylin and eosin stain. Ziehl-Neelsen was used for demonstration of acid-fast bacilli. A final pathological and/or a clinical was reached in each case. Serum adenosine deaminase, angiotensinconverting enzyme, C-reactive protein, procalcitonin, and tumor markers were obtained when indicated. Tuberculine test was performed in patients with a suspected tuberculous pleural effusion.

A minimum of $200 \mathrm{ml}$ pleural fluid was obtained from each patient for differential cell count and exfoliative cytological analysis. After pleural fluid was centrifuged for 10 minutes at $2000 \mathrm{rpm}$, the smears were prepared from the remaining sediment. Giemsa, hematoxylin eosin, and Papanicolaou were used for staining cytologic smears. For bloody fluids, 0.1 N HCL was used as an hemolyzing agent. Smear and culture for microbiological analysis of the pleural fluid were done to identify infection. Ziehl-Neelsen stain was used to detect acid-fast bacilli. Statistical analysis was performed by calculating the sensitivity and the specificity within a $95 \%$ confidence interval. Correlation of the pleural cytology findings was done with the final clinical diagnosis confirmed by histopathologic biopsy results. Sensitivity and specificity of the exfoliative cell cytology was calculated within $95 \%$ confidence intervals in regard to the final diagnosis for exudative pleural effusions.

\section{Results}

A total of six hundred patients, 420 males (70\%) and 180 females were included in the study. The age of the patients ranged from 18 to $78(42.8 \pm 14.2)$ years. Exfoliative cytology of the pleural fluid was diagnostic in 354 (76.4\%) patients while cytology predicted the final diagnosis in 78 (13.0\%) subjects. Final diagnosis was confirmed by histopathologic examination of the closed pleural, thoracoscopic, bronchoscopic, mediastinoscopic, lymph node, pulmonary, and extrapulmonary organ biopsy samples. All subjects tolerated the thoracentesis well. Vasovagal reactions occurred in 42 (7\%) of the subjects. Pneu- mothorax developed in 38 (6.3\%) patients and in 19 (3.1) \% chest tube was inserted.

Correlation of exfoliative pleural fluid cytology diagnosis was done in regard to the final clinical diagnosis. The final diagnosis of the pleural effusion was reached according to the evaluation of the histopathologic tissue obtained by the lymph node biopsy, lung biopsy, extrapulmonary organ biopsy, closed or thoracoscopic biopsy sampling. Final diagnosis of the pleural fluid etiology is shown in Table 1.

Cytologic examination predicted the final diagnosis in 80 (13.3\%) patients and led the clinician in the right diagnostic pathway. Of these, 52 were malignant, 18 were tuberculous, and 10 were infectious pleural effusions. The overall cytology sensitivity was $76.4 \%$, positive in 354 patients. The second and the third pleural fluid samples were positive in 24 (4\%) and 18 (3\%) patients, respectively. When cytology was combined with the clinical data including the laboratory (serum biochemistry, ADA, ACE) and radiology (CT, PET/CT, MR, etc.) results, the overall sensitivity was higher than cytology alone (Table 1) and reached to a $80.9 \%$ diagnostic ratio for all the patients.

Total white cell count and differential analysis were useful to characterize and diagnose the cause of a pleural effusion by identifying the predominant cell type. Neutrophil-rich effusions were often encountered in parapneumonic effusions and pleural effusions due to pulmonary embolism, viral infection, acute tuberculosis, or benign asbestos-related pleural disease. Lymphocytic effusions were mostly found in tuberculosis, primary or metastatic neoplasia, and collagen vascular disease. An eosinophilic effusion (>10\% cell count) was frequent in neoplasia but also occurred in pleural effusions associated with infection, pulmonary infarction, Churg-Strauss syndrome, and benign asbestos-related pleural disease. The most common cause of hemorrhagic pleural effusions was malignancy and tuberculosis. Malignant effusions were hemorrhagic in $28 \%$ of the patients.

The highest diagnostic yield of exfoliative cytology was obtained for malignant effusions followed by pleural effusions due to tuberculosis, infection and collagen vascular diseases. Malignancy was confirmed by cytology alone in $240(240 / 342,70.1 \%)$ malignant pleural effusions. Of the 342 malignant pleural effusions, 164 (174/342, 50.9\%) cases were diagnosed as metastatic adenocarcinoma which was the most frequent cell type followed by small cell, and squamous carcinoma among patients with metastatic pleural involvement. The distinct features of a single cell or a group of cells were the diagnostic hallmark of the malignant pleural effusions. Malignant cells appeared as individual, sheets of cohesive cells, and spherical clusters. Cells with enlarged nuclei, coarse chromatine, prominent nuclei, and atypical mitoses were frequent. Adenocarcinoma cells were either single, formed clusters, or adenoid groups with a pale vacuolated cytoplasm while papillary or acinar structures were dominant (Figure 1). Sixty-eight (68/600, $11.3 \%$ ) pleural effusions were hemorrhagic. Metastatic small cell carcinoma cells were seen in the pleural fluid samples of the 82 patients

Table 1. Final diagnosis of patients with an exudative pleural effusion.

\begin{tabular}{lcc} 
Etiology of exudative pleural effusions & Final diagnosis n/total & Percentage \\
Malignant pleural effusion & $342 / 600$ & $57 \%$ \\
\hline Tuberculous pleural effusion & $90 / 600$ & $15 \%$ \\
\hline Parapneumonic pleural effusion & $50 / 600$ & $8.3 \%$ \\
\hline CVD pleural effusion & $28 / 600$ & $4.6 \%$ \\
\hline Miscellaneous pleural effusion & $30 / 600$ & $5 \%$ \\
\hline Undiagnosed pleural effusion & $60 / 600$ & $10 \%$ \\
\hline
\end{tabular}

n: number of patients; CVD: collagen vascular disease. 
(82/342, 25.3\%). The fluid was hemorrhagic in 39 (39/342, 11.4\%) patients. The cells appeared discretely or in loose cohesive clusters of small atypical epithelial cells like lymphocytes, all with large dense nuclei, coarse chromatine, inconspicuous nucleoli, and scant cytoplasm. Squamous cells were present in 41 samples $(41 / 324,12.6 \%)$ and eighteen samples (18/342, 5.3\%) were hemorrhagic. The squamous cells were discrete, had usually orangeophilic cytoplasm, and showed evidence of keratinization with coarse chromatin. The cells tended to have a flatter and two-dimensional appearance than those of adenocarcinoma. Diagnostic yield increased to $92.3 \%$ (295/342) when cytology was combined with other laboratory and radiology methods in malignant effusions. The primary site of malignancy was identified in 194 patients (56.7\%) when exfoliative cytology (Figure 2) and other laboratory findings were collaborated. Forty-six (13.4\%) of the exudative effusions were positive for hematologic malignancies like lymphoma and leukemia. Cell population in lymphoma effusions consisted of single, large cells, round to irregular nuclei, prominent irregular nucleoli, coarse chromatin, scant to abundant cytoplasm. Malignant mesothelioma was diagnosed in 18 patients (5.2\%) by exfoliative cytology. These effusions were exudative and had a viscous appearance. The mesothelial cells varied considerably in size, were arranged as single cells or in sheets, clusters, morulae or papillary fashion forming large clusters with lumpy borders, and lacked the significant degree of cytological pleomorphism. The sensitivity (56.8\%) and the specificity
(52.4\%) of exfoliative cytology for malignant mesothelioma were low in our study.

The final diagnosis was tuberculosis in 90 patients. Sensitivity of exfoliative cytology alone was $76.4 \%$ while the sensitivity increased to $86.7 \%$ when cytology was collaborated with other clinical findings (Table 2). Almost all tuberculous effusion samples were rich in small lymphocytes ranging from $68 \%$ to $94 \%$ of the total cell count. The effusions also contained small numbers of neutrophils, macrophages, plasma cells or red blood cells. Pleural effusion was hemorrhagic in 20 $(20 / 90,22.2 \%)$ patients. In none of the tuberculous pleural effusions, the eosinophil count was more than $10 \%$ or the mesothelial cell ratio was over $1 \%$ of the total cells. The diagnostic yield of cytology was $69.4 \%$ for parapneumonic pleural effusions. Sensitivity increased up to $87.2 \%$ when exfoliative cytology and other laboratory findings (Table 2) were collaborated. Most of the cells were neutrophils. A high proportion of neutrophils had undergone degeneration appearing as smudgy anucleated blobs while the mean percentage of neutrophil ratio was over $68 \%$ in parapneumonic pleural effusions. Microorganisms were also frequently (71.8\%) encountered either in dispersed fashion or in cloudlike colonies.

Pleural effusion due to collagen vascular diseases was diagnosed by exfoliative cytology in $12(12 / 28,42.8 \%)$ of the 28 patients. Of these 28 patients, eighteen had SLE while ten had rheumatoid pleural effusions. LE effusions were characterized by predominance of lymphocytes or

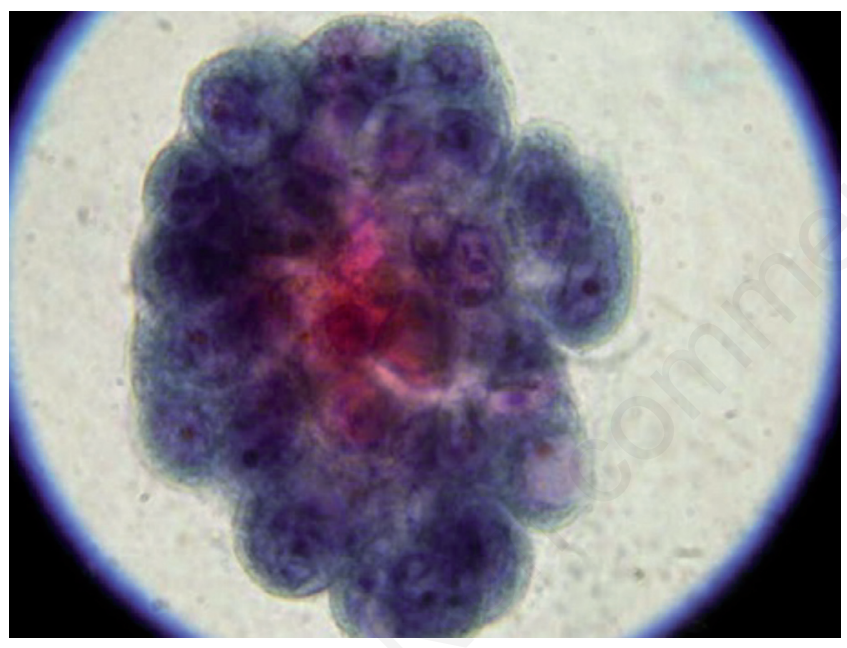

Figure 1. Metastatic cells from pulmonary adenocarcinoma with a vacuolated cytoplasm forming adenoid groups (Papanicolaou stain; $\mathbf{x} 400$ ).

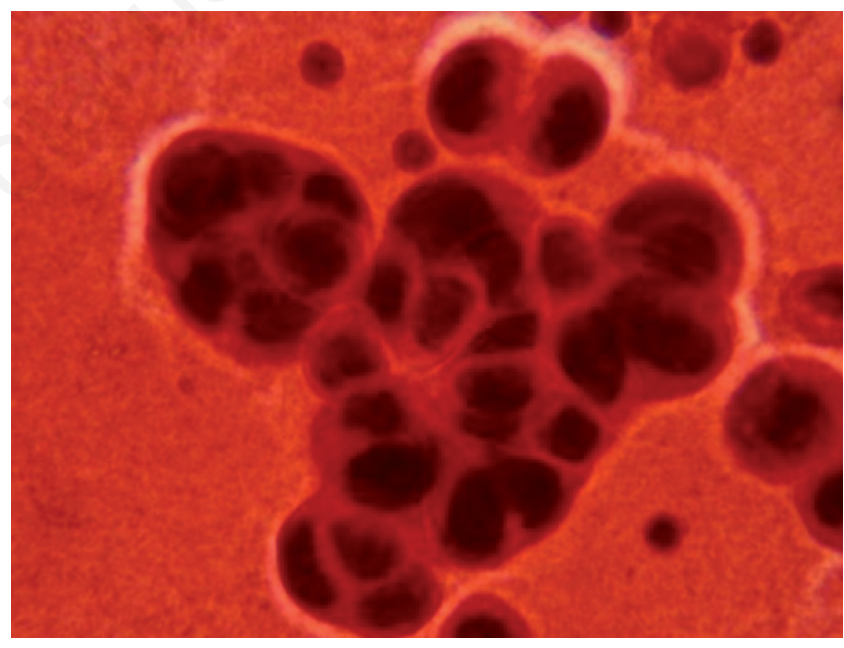

Figure 2. Hyperchromatic adenocarcinoma cells with enlarged nucleus from breast showing a glandular pattern (Papanicolaou stain; $x 400$ ).

Table 2. Sensitivity of pleural fluid cytology for malignancy, tuberculosis, collagen vascular disease, and infection.

\begin{tabular}{lccccc} 
& Cytology Dx/total & Sensitivity (\%) & CI (95\%) & Specificity (\%) & CI (95\%) \\
Total Dx by cytology & $348 / 600$ & 76.4 & $71.2-79.8$ & 62.5 & $64.3-78.2$ \\
Total Dx by cytology+clinical findings & $436 / 600$ & 80.9 & $78.2-81.4$ & 82.3 & $78.4-84.8$ \\
\hline Malignancy Dx by cytology & $240 / 342$ & 70.1 & $72.2-81.6$ & 87.8 & $76.4-89.6$ \\
Malignancy Dx by cytology+clinical findings & $295 / 342$ & 92.3 & $91.2-98.2$ & 97.4 & $81.6-98.1$ \\
\hline Tuberculosis Dx by cytology & $65 / 90$ & 76.4 & $72.6-82.0$ & 60.2 & $60.1-79.8$ \\
Tuberculosis Dx by cytology+clinical findings & $82 / 90$ & 86.7 & $78.0-88.1$ & 90.2 & $84.2-92.4$ \\
\hline Infection Dx by cytology & $31 / 50$ & 69.4 & $72.8-80.4$ & 63.6 & $62.1-71.6$ \\
Infection Dx by cytology+clinical findings & $41 / 50$ & 87.2 & $82.6-92.6$ & 66.7 & $64.2-76.1$ \\
\hline CVD Dx by cytology & $12 / 28$ & 42.8 & $72.4-79.8$ & 50.0 & $54.2-68.6$ \\
CVD Dx by cytology+clinical findings & $24 / 28$ & 96.0 & $82.9-92.2$ & 50.0 & $52.4-70.4$ \\
\hline
\end{tabular}

CI: confidence interval; Dx: diagnosis; CVD: collagen vascular disease. 
neutrophils. Lupus erythematosus cells were encountered in five patients. Rheumatoid pleural effusions were characterized by large elongated, giant round, or oval nucleated cells. The number of giant multinucleated macrophages with acidophilic cytoplasm were abundant in a background of amorphous granular material. The granular necrotic background formed by macrophages dominated the smear. Rheumatoid arthritis cells were observed in four samples. Mesothelial cells were virtually absent in rheumatoid effusions.

The incidence of pneumothorax due to thoracentesis was $6 \%$ in our patients while no major complications occurred. The initial exfoliative cytologic examination predicted the final diagnosis in $13.2 \%$ of the patients when it was not diagnostic by itself. The predictive value and the likelihood ratio of the exfoliative cytology is depicted in Table 3. Benign pleural effusions contained mesothelial cells, histiocytes, and polymorphonuclear leukocytes in varying proportions with common binucleation or multinucleation but with rare cytoplasmic vacuoles. These effusions usually did not reveal any of the specific or exclusive features of the metastatic malignant cells.

\section{Discussion}

Exudative pleural effusions arise from the pathologic processes relevant to the serosal membranes. Exfoliative pleural effusion cytology with differential cell count is a useful and a frequently used initial step for the diagnosis of exudative pleural fluids [2]. Cytologic analysis represents the whole surface of the pleura because it has the advantage of exfoliated cells in the fluid representative of a much larger surface than the pleural biopsy [4]. The major limitation of the procedure is the false positive or negative results. Sixty to seventy percent of metastatic malignancies to the pleura and $30-40 \%$ of malignant mesotheliomas can be diagnosed by exfoliative cytology [3,5,6]. Even if the initial cytological examination is not diagnostic, exfoliative cytology and differential cell count lead the clinician in the right diagnostic pathway for final diagnosis [2]. The results of our study revealed that cytologic examination established the highest diagnostic yield in adenocarcinoma followed by tuberculosis. On the other hand, the differential cell count of polymorphonuclear leukocytes led the clinician in the accurate track by pointing out to specific diseases and narrowed the differential diagnosis in our study. In equivocal cases where exfoliative cytology was not adequate for diagnosis, the collaboration of other laboratory and radiologic methods such as serum biochemistry, tuberculin test, ADA, CT, and PET/CT with exfoliative cytology further increased the diagnostic yield of this simple method. Cytology proved to be the initial diagnostic hallmark for the assessment of exudative pleural effusions.
Exfoliative cytology alone was diagnostic positive in $70.1 \%$ of our patients with an exudative pleural effusion. If three separate pleural fluid specimens are submitted to an experienced cytologist, nearly $80 \%$ of the patients will have positive results by cytology alone [7,8]. The first sample was diagnostic in $60.4 \%$ of the patients while the analysis of the second and third samples increased the sensitivity to $65.8 \%$ and to $70.1 \%$ in our study, respectively. The diagnostic rates of cytology alone were higher than the Bueno and Jarvi $[3,8]$ studies. This may be due to the etiology, distinctive tumor cell types, the tumor load in the pleural space, and to the skill of the cytologist. The highest final diagnostic results were obtained in malignant pleural effusions followed by tuberculosis. Most of the metastatic adenocarcinomas were identified with cytologic examination alone while the diagnostic yield was less for small cell, squamous cell carcinomas, Hodgkin's disease, mesothelioma, and sarcoma. In patients with a malignant pleural effusion, exfoliative cytology of the pleural fluid proved to be an efficient and a minimally invasive procedure for the final diagnosis.

Cytologic diagnosis of malignant effusions has been reported to be between $40 \%$ and $87 \%$ in different studies [1,7,8]. Several factors may influence the sensitivity of cytology in malignant effusions. The effusion may develop secondary to other factors such as infection, pulmonary emboli, or lymphatic blockade. Positive cytology may depend upon the tumor type as cytology is more frequently positive in adenocarcinomas. If cell blocks are used the diagnostic yield becomes greater. The incidence of positive pleural cytology is higher in patients with a large tumor burden in the pleural space $[3,5,9]$. The overall sensitivity of pleural cytology was $76.4 \%$ for exudative pleural effusions, reaching to $80.9 \%$ if the cytologic examination was integrated with other laboratory findings in our study. Exfoliative cytology was diagnostic in $70.1 \%$ of the malignant effusions while the highest diagnostic yield was obtained in adenocarcinomas which was compatible with the previous studies. When exfoliative cytology, clinical, and laboratory findings were collaborated the diagnostic accuracy increased to $92.3 \%$ for malignant effusions. The primary site for metastatic pleural effusions was identified with confidence in $72.8 \%$ of the patients when the clinical and the radiologic findings were collaborated with exfoliative cytology. The high diagnostic yield obtained in malignant pleural effusions by exfoliative cytology is related to the existence of specific cells and to the presence of significant alterations in the structure of malignant cells in these effusions. Tight intercellular cohesion of epithelial tumors leads to shedding and exfoliation of malignant cells into the pleural effusion. This cellular structure may explain the enhanced the sensitivity of cytology for such tumors. The lowest diagnostic yield of exfoliative cytology was achieved for malignant effusions due to lymphoma, sarcoma, and mesothelioma. In non-epithelial tumors, cellular

Table 3. Predictive value and likelihood ratio of exfoliative cytology.

\begin{tabular}{lccccccccc} 
& n/total & PPV & CI (95\%) & NPV & CI (95\%) & LR+ & CI (95\%) & LR- & CI (95\%) \\
Total Dx by cytology & $354 / 600$ & 95.9 & $72.8-78.1$ & 18.7 & $71.6-76.9$ & 3.2 & $70.4-75.6$ & 0.31 & $71,1-74.4$ \\
Total Dx by cytology+clinical findings & $442 / 600$ & 97.5 & $76.2-80.4$ & 33.7 & $75.4-81.2$ & 4.2 & $74.2-82.3$ & 0.24 & $76.3-80.9$ \\
\hline Malignancy Dx by cytology & $240 / 342$ & 97.8 & $74.1-81.2$ & 37.1 & $71.3-80.4$ & 3.9 & $72.7-81.3$ & 0.25 & $70.4-75.2$ \\
Malignancy Dx by cytology+clinical findings & $295 / 342$ & 99.6 & $79.4-86.2$ & 82.6 & $78.6-82.7$ & 36 & $76.1-83.2$ & 0.028 & $78.3-82.4$ \\
\hline Tuberculosis Dx by cytology & $65 / 90$ & 97 & $73.2-80.6$ & 13 & $70.4-81.6$ & 3.2 & $75.1-80.4$ & 0.30 & $74.2-81.2$ \\
Tuberculosis Dx by cytology+clinical findings & $82 / 90$ & 98.9 & $76.8-82.1$ & 39.1 & $74.2-81.4$ & 6.5 & $73.8-82.6$ & 0.15 & $81.2-86.3$ \\
\hline Infection Dx by cytology & $27 / 50$ & 77.1 & $70.6-76.8$ & 46.7 & $72.3-77.1$ & 2.24 & $71.2-76.5$ & 0.44 & $77.3-82.5$ \\
Infection Dx by cytology+clinical findings & $41 / 50$ & 97.6 & $71.2-79.2$ & 25 & $70.4-78.3$ & 6.8 & $72.3-77.1$ & 0.15 & $72.7-81.8$ \\
\hline CVD Dx by cytology & $12 / 28$ & 90 & $72.2-81.4$ & 25 & $70.6-80.6$ & 3.0 & $72.7-81.2$ & 0.33 & $70.2-81.7$ \\
CVD Dx by cytology+clinical findings & $24 / 28$ & 96 & $73.2-80.2$ & 50 & $72.3-81.4$ & 24 & $70.3-80.8$ & 0.41 & $73.1-79.1$ \\
\hline
\end{tabular}

n: number of patients; Dx: diagnosis; CVD: collagen vascular disease; CI: confidence interval; PPV: positive predictive valute; NPV: negative predictive value; LR: likelihood ratio.. 
dyscohesion may be the primary cause leading to diminished shedding and exfoliation of malignant cell into the effusion that has declined the diagnostic yield of exfoliative cytology. The low diagnostic yield of pleural cytology in lymphomas may also be due to the fact that the effusion is usually due to the thoracic duct obstruction rather than the pleural infiltration.

In sarcomas, the low diagnostic accuracy of cytology is relevant to the solid tumor nature and thereby the lack of cell shedding into the pleural space. The low sensitivity of cytology for mesothelioma is related to tumor nature, lack of mesothelioma cells shedding into the pleural space, and the similar features of benign reactive mesothelial cells with the malignant cells. The dyscohesive nature of tumor cells in these nonepithelial tumors may have further prevented the shedding of malignant cells into the fluid. No diagnosis is ever established in approximately of $15 \%$ of patients with exudative effusions [6,10,11]. Even after thoracoscopy $10 \%$ of the pleural effusion may remain undiagnosed [12-14]. In our study, the incidence of undiagnosed pleural effusions was $10 \%$ and was compatible with the previous researches. Another aspect of our study was that pleural fluid cytology led the clinician in the right diagnostic pathway for further evaluation even if it were not diagnostic on its own initially by predicting the final diagnosis in $14.8 \%$ of the effusions. The incidence iatrogenic pneumothorax was low in our patients while almost all the patients tolerated the procedure well without any major complications which appeared to be another advantage of this clinical tool compared to other invasive diagnostic modalities.

A tuberculous pleural effusion is a diagnostic challenge for the pulmonary clinician. It is impossible to differentiate a tuberculous effusion from a malignant pleural effusion on clinical grounds alone and usually more invasive diagnostic interventions are needed for diagnosis. The absence of mesothelial cells along with the presence of more than $60 \%$ small lymphocytes were noted to be a strong evidence for tuberculosis in our study. The absence of mesothelial cells in tuberculous pleural effusions is attributable to the deposition of fibrin on the pleural surface, either sealing off the mesothelial cells, destroying them, or both $[3,15]$. The fibrotic chronic clinical sequela of tuberculous effusions may have further prevented the shedding of mesothelial cells into the effusion. Although the mechanism and control of eosinophil accumulation in the pleural space is unknown, they play an important role in idiopathic, allergic diseases, drug reactions, or the presence of air in the pleural space while tuberculous pleural effusions rarely contain more than $10 \%$ eosinophils [16-18]. The presence of numerous mesothelial cells or eosinophils in the pleural fluid samples was useful to exclude tuberculosis in the differential diagnosis of exudative pleural effusions.

The diagnostic sensitivity of pleural cytology for pleural effusions associated with collagen vascular diseases was lower than that of other exudative pleural effusions. This finding is attributed to the lack of specific cells, absence of specific cell patterns in these effusions, and to the scarcity of cells due to the intensive inflammation with combined fibrosis in such effusions. Deposition of fibrin on the pleural surfaces may have promoted the lower diagnostic yield by loss of cell shedding into the fluid. The diagnosis for collagen vascular disease effusions was essentially clinical. Collaboration of clinical data and laboratory findings with pleural cytology increased the diagnostic yield reaching the highest values in our study compared to other diseases while cytology alone revealed the lowest diagnostic outcome among other pleural effusions. Exfoliative pleural fluid analysis was not diagnostic in $25.4 \%$ alone and in $19.1 \%$ when collaborated with other laboratory findings in such patients.

There are some limitations of our study. The patient population may be considered as small. Genetic as well as environmental factors were not evaluated because patients included in this study consisted of only
Caucasian people. The study was performed to assess exclusively the diagnostic yield conventional exfoliative cytology. We did not include cell blocks, immunohistochemistry studies, lymphocyte subset analysis, and flow cytometry in this research. The sensitivity and the specificity of exfoliative cytology would reach to much higher levels if the aforementioned investigations had been included in the study. The small number of patients with collagen vascular diseases may have led to or influenced the low diagnostic yield of cytology in such type of effusions. Studies comprising higher patient populations with more heterogenous clinical profiles may further enlighten the utility of exfoliative cytology in exudative pleural effusions.

Exfoliative cytology of the pleural fluid is a definitive crucial initial diagnostic step in the evaluation of exudative pleural effusions. This simple non-invasive technique may be considered as the best fundamental diagnostic bench in the hands of an experienced cytologist without any serious complications reaching to a high diagnostic yield. Pleural fluid cytology can narrow the differential diagnosis considerably and indicate the next step leading the clinician in the correct pathway for final diagnosis even when not it is diagnostic on its own precluding the unnecessary invasive interventions. In conclusion, cytologic analysis of pleural fluid may identify the final diagnosis in exudative pleural effusions with an extremely high diagnostic yield.

\section{Conclusions}

Exfoliative pleural cytology is a simple and a highly sensitive diagnostic modality especially for malignant and tuberculous effusions that may be a diagnostic challenge for the clinician. The diagnostic sensitivity may be attributable to the presence of cell population representative of a much larger pleural surface than biopsy for malignant pleural effusions. In benign effusions, the diagnostic yield of exfoliative cytology is low due to the absence specific cells or structural cell alterations. Combined exfoliative cytology and laboratory findings may further increase the final diagnostic yield of cytology. Cytologic features of malignant cells may establish the primary site in metastatic effusions when collaborated with other clinical and laboratory findings. Exfoliative cytology of the pleural fluid is not only useful in reaching the final diagnosis but also points out the possible etiologies by predicting the final diagnosis in advance when it is not diagnostic own its own thereby leading the clinician to an accurate diagnostic pathway. Other advantages of cytologic analysis include low incidence of complications of thoracentesis and the ease of reaching diagnosis without further redundant invasive interventions.

\section{References}

1. Kushwaha R, Shashikala P, Hiremath S, Basavaraj HG. Cells in pleural fluid and their value in differential diagnosis. J Cytol 2008;25:138-43.

2. Tetikkurt C, Kara YB, Tetikkurt S, et al. The value of cytology in the diagnosis of pleural effusions. Brit J Med Medic Res 2014;):2203-11.

3. Jarvi OH, Kunnas RJ, Laitio MT, et al. The accuracy and significance of cytologic cancer diagnosis of pleural effusions. Acta Cytol 1972;16:152-7.

4. Sherwani R, Akhtar K, Naqvi H, et al Diagnostic and prognostic significance of cytology in effusions. J Cytol 2005;22:73-7.

5. Light RW, Erozan YS, Ball WC. Cells in pleural fluid: their value in differential diagnosis. Arch Intern Med 1973;132:854-60.

6. Ong KC, Indumathi V, Poh WT, et al. The diagnostic yield of pleural cytology in malignant pleural effusions. Singapore Med J 2000;41:19-23. 
7. Dekker A, Bupp PA. Cytology of serous effusions. An investigation into the usefulness of cell blocks versus smears. Am J Clin Pathol 1978;70:855-60.

8. Bueno CE, Clement G, Castro BC, et al. Cytologic and bacterial analysis of fluid and pleural biopsy specimens with Cope's needle. Arch Intern Med 1990;150:1190-4.

9. Filie AC, Copel C, Wilder AM, et al. Individual specimen triage of effusion samples: an improvement in the standard of practice, or a waste of resources? Diagn Cytopathol 2000;22:7-10.

10. Light RW. Approach to the patient. In: RW Light, editor. Pleural diseases. Philadelphia, Lippincott Williams and Wilkins; 2013; pp 128-39.

11. Kendall SW, Bryan AJ, Large SR, et al. Pleural effusions: is thoracoscopy a reliable investigation? A retrospective review. Respir Med 1992;86:437-40.
12. Ferrer J, Roldan J, Teixidor J, et all. Predictors of pleural malignancy in patients undergoing thoracoscopy. Chest 2005;127:1017-22.

13. Loddenkemper R, Boutin C. Thoracoscopy: diagnostic and therapeutic indications. Eur Respir J 1993;6:1544-55.

14. Argento AC, Wahidi MM. Undiagnosed pleural effusions. In: WR Kight and YCG Lee, editors. Textbook of pleural diseases. New York, CRC Press; 2016; pp 456-63.

15. Kalomenidis J. New advances in the investigation of pleural diseases. Pneumologie 2003;16:247-51.

16. Hurwitz S, Leiman G, Shapiro C. Mesothelial cells in pleural fluid: TB or not TB? S Afr Med J 1980;57:937-9.

17. Epstein DM, Kline RM, Albelda SM, Miller WT. Tuberculous pleural effusions. Chest 1987;91:106-9.

18. Light RW. Establishing the diagnosis of tuberculous pleuritis. Arch Intern Med 1998;158:1967-8. 